\title{
Nordiques
}

35 | 2018

Tove Jansson : Par delà les genres. Pratiques

linguistiques dans le Norden du XXIe siècle : Quels

enjeux sociaux?

\section{Oslo : une ville, deux dialectes?}

\section{Karine Stjernholm}

Traducteur : Sarah Harchaoui

\section{(2) OpenEdition}

Journals

Édition électronique

URL : https://journals.openedition.org/nordiques/1014

ISSN : 2777-8479

Éditeur :

Association Norden, Bibliothèque de Caen la mer

Édition imprimée

Date de publication : 1 mai 2018

Pagination : 117-134

ISBN : 979-1-0959140-1-3

ISSN : $1761-7677$

\section{Référence électronique}

Karine Stjernholm, « Oslo : une ville, deux dialectes? », Nordiques [En ligne], 35 | 2018, mis en ligne le 02 février 2021, consulté le 06 octobre 2021. URL : http://journals.openedition.org/nordiques/1014 


\section{Oslo : une ville, deux dialectes ?}

Karine Stjernholm*

\section{RÉSUMÉ}

Cet article présente une analyse comparative de deux chansons contemporaines de rap à Oslo dans lesquelles la variation linguistique est utilisée en tant qu'outil littéraire et marqueur sociogéographique. La capitale norvégienne a historiquement connu deux variétés orales, qui ont convergé au cours des quatre cents dernières années. Ce processus a abouti à une situation orale où ces variétés, de nos jours, étroitement liées, sont en confrontation à la fois socialement et linguistiquement. L'article expose les principales différences linguistiques entre ce qu'on appelle la variété ouest (issue de la variété haute du danois parlée par les classes sociales supérieures au début du XXe siècle), et la variété est (issue de l'ancienne variété basse parlée par la population autochtone d'Oslo). Aujourd'hui, il est difficile de les classer comme deux variétés distinctes, car elles partagent une base linguistique commune, malgré quelques différences phonologiques, morphologiques et syntaxiques qui subsistent. Ces différences restent néanmoins exploitées par les locuteurs pour négocier une identité sociale - et dans le contexte d'Oslo également géographique.

\section{ABSTRACT}

The article presents an analysis of two contemporary rap lyrics from Oslo, the capital of Norway, where linguistic variation is used as a literary device. Oslo historically had two speech varieties that have converged in the past 400 years. This process has resulted in a speech situation where these now closely related varieties are contesting both socially and linguistically. The article presents the main linguistic differences between what is called the Western and Eastern speech variety in Oslo. The Western variety is a descendant of the Danish high variety spoken in higher social classes in Oslo around the beginning of the $20^{\text {th }}$ century. The Eastern variety is a former low variety spoken among Oslo's simultaneous autochthon population. Today it is difficult to classify these as two separate varieties. They share a common linguistic basis, even if both phonologic, morphologic, and also some syntactic features still separate them. This remaining variation is something the speakers of these varieties exploit to negotiate social - and in this context also geographic - identity. The analysis of the rap lyrics shows an example of how this negotiation is being practiced.

* Karine Stjernholm est docteur en linguistique et maitre de conférences à la Høyskolen d'Østfold en Norvège. Ses intérêts en recherche se situent autour des questions de sociolinguistique urbaine, du multilinguisme et du paysage linguistique norvégien contemporain.

1 Une partie du contenu de cet article a été publiée dans mon exposé liminaire lors ma soutenance de thèse de doctorat à Oslo le 19 juin 2013. Un article plus théorique fera l'objet d'une publication en 2019 (Karine Stjernholm, "Variasjon som virkemiddel : oslomål i praksis », Norsk lingvistisk tidsskrift, 1, à paraitre). Un grand merci à Sarah Harchaoui qui m’a invitée aux Journées nordiques à l'université ParisSorbonne au printemps 2017 et qui s'est chargée de la publication des communications de ces Journées. Je dois également un grand merci à mon cher collègue Marcus Axelsson de la Høyskolen d'Østfold pour m’avoir inspirée et surtout pour m’avoir indiqué la bonne terminologie linguistique à utiliser. 


\section{INTRODUCTION}

Oslo, capitale et première ville de Norvège, présente traditionnellement deux variétés parlées, celle de l'ouest, aujourd'hui appelée vestvarieteten, et celle de l'est, intitulée østvarieteten ${ }^{2}$. Les récentes recherches menées sur le sujet semblent indiquer que ces deux variétés sont actuellement en train de converger.

La variété de l'ouest, appelée " den dannede dagligtale " " la langue courante éduquée "), est héritière du danois, et était parlée par les classes supérieures dans les centres urbains - tandis que la variété de l'est est construite à partir des dialectes norvégiens présents dans la région de l'Østlandet avant l'arrivée des Danois. Dès le début du XXe siècle, le linguiste Amund B. Larsen (1907) atteste déjà de la convergence, voire de la fusion de ces deux variétés.

La convergence entre l'østkantmål (« le parler des quartiers est ») et le vestkantmål (« le parler des quartiers ouest ») est illustrée par l'image suivante :

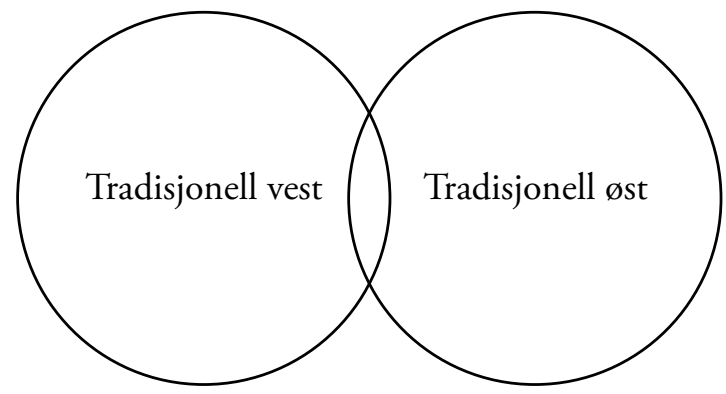

Image 1 - L’østkantmål et le vestkantmål à Oslo se recoupent peu traditionnellement.

Même si ces variétés convergent et sont aujourd'hui perçues comme une seule et même variété, cette dernière présente une importante variation étroitement liée à la scission historique de la ville d'Oslo entre les quartiers est et ouest.

Dans cet article, mon analyse portera sur les paroles de deux chansons ayant comme thématique la ville d'Oslo et démontrera comment cette variété est actuellement exploitée. La première chanson, intitulée "Nilsen » (2013) et interprétée par les artistes Don Martin et Tommy Tee, est représentative des quartiers est de la ville. La seconde, intitulée "Bærumsgrammatik » (2004), est réalisée par les artistes Erik et Kriss (Erik Mortvedt et Kristoffer Tømmerbakke). Malgré sa position géographique située à l'extérieur d'Oslo, Bærum est rattaché au GrandOslo pour des raisons socioculturelles ${ }^{3}$.

2 Karine Stjernholm, Stedet velger ikke lenger deg, du velger et sted, thèse de doctorat, Institut d'études linguistiques et nordiques, Université d'Oslo, 2013.

3 Ibid. 


\section{HiP-HOP ET ANCRAGE LOCAL}

Dans la culture hip-hop, et particulièrement afro-américaine, affirmer son appartenance à une localité est un facteur primordial. L'authenticité du hip-hop vient d'ailleurs de son ancrage local, où les traits dialectaux locaux " ... have become the "base languages" of local hip-hop scenes as well a key marker of local authenticity" ${ }^{4}$.

Le caractère innovant et transgressif du langage hip-hop doit être envisagé dans un contexte de construction de nouveaux lectes (variétés), dans lequel ce qui est innovant provient de l'innovation linguistiques. Dans cet article, nous nous focaliserons sur la manière dont les textes de rap expriment l'affiliation locale à l'østkant et le vestkant à Oslo.

L'expression de cette appartenance locale peut également être perçue comme une forme de "glocalisation » ou de réorientation vers le local, qui selon Barbara Johnstone ${ }^{6}$ doit être comprise comme une conséquence directe de la mondialisation. Celle-ci a encouragé la mobilité sociale et géographique, qui à son tour a augmenté le contact entre les dialectes et leur nivellement. Toujours selon Barbara Johnstone ${ }^{7}$, ce processus " ... may spark the kinds of metapragmatic activities that lead to ideological differentiation among dialects ". En d'autres termes, la convergence des dialectes a pour conséquence immédiate le besoin de manifester et de signaler son appartenance dialectale.

\section{LA DIVISION D'OSLO : ARRIÈRE-PLAN}

Après la révolution industrielle du XIX ${ }^{\mathrm{e}}$ siècle, les premières usines à Oslo se sont établies le long de la rivière Akerselva qui traverse et scinde la ville en deux ${ }^{8}$. Ce fait historique permet d'expliquer pourquoi Oslo a été, et demeure, une ville divisée entre est et ouest.

La rivière fournissait de l'énergie aux usines, et les ouvriers se sont logiquement installés le plus près de leurs lieux de travail. À cette époque, la ville se caractérise par une forte croissance démographique, les nouveaux habitants étant principalement originaires de l'Østlandet'. Cela laisse supposer que les ouvriers parlaient

4 Cecelia Cutler, Unn Røyneland, "Where the fuck am I from ?: Hip-Hop youth and the (re) negotiation of language and identity in Norway and the US ", in Language, Youth and Identity in the $21^{\text {st }}$ Century. Linguistic Practices across Urban Spaces, J. Nortier et B. A. Svendsen (dir.), Cambridge, Cambridge University Press, 2015, p. 42.

5 Jan Sverre Knudsen, "Playing with Words as if it was a Rap Game : Hip-Hop Street Language in Oslo ", in Multilingual Urban Scandinavia. New Linguistic Practices, P. Quist et B. A. Svendsen (dir.), Bristol, Multilingual Matters, 2010, p. 157.

6 Barbara Johnstone, "Indexing the Local ", in The Handbook of Language and Globalization, N. Coupland (dir.), Malden, Wiley-Blackwell, 2010, p. 387.

7 Barbara Johnstone, op. cit., p. 391.

8 Karine Stjernholm, op. cit., p. 7.

9 Jan Eivind Myhre, Hovedstaden Christiania. Oslo bys historie, Oslo, Cappelen, 1990, t. III, p. 23. 
majoritairement des dialectes de l'est du pays, qui sont à l'origine de la formation de l'østkantmålet à Oslo. Amund B. Larsen ${ }^{10}$ souligne que le parler des quartiers est (ou " la langue vulgaire ", litt. vulgarsproget) a des liens plus étroits avec Østre Aker (la partie est de la rivière) que Vestre Aker (la partie ouest).

Les classes sociales supérieures occupaient l'ouest de la ville et parlaient danois avant d'utiliser la " langue courante éduquée " ${ }^{11}$, c'est-à-dire une langue orale norvégienne fortement influencée par des traits de la langue orale danoise, et encore plus par le danois écrit, qui durant la période servait de langue écrite en Norvège.

Ce schéma d'urbanisation caractérise encore de nos jours la ville d'Oslo. Dans les quartiers de l'ouest, la population possède toujours un niveau supérieur concernant l'éducation, les revenus, le logement et la santé. Ces différences sont aujourd'hui renforcées par le fait que l'ouest montre un taux d'immigration significativement plus faible que dans les quartiers de l'est ${ }^{12}$.

Outre une immigration importante, Oslo connait une forte migration interne ${ }^{13}$. L'image ci-dessous illustre les vagues migratoires en Norvège en 2009. Il est à noter que la pression sur l'Østlandet et Oslo est considérable.

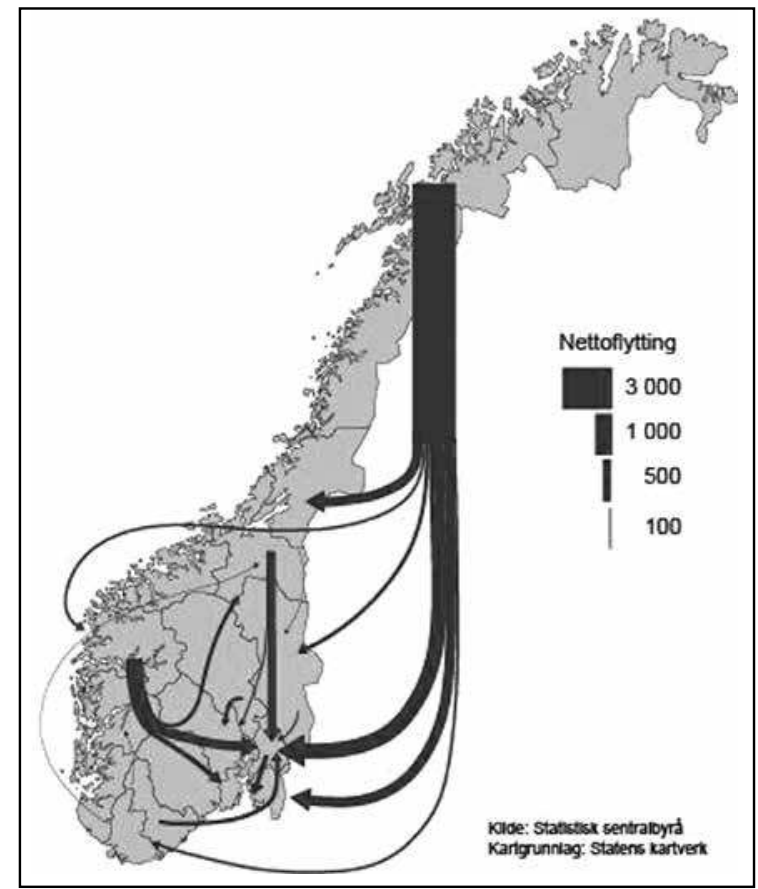

Image 2 - Vagues migratoires en Norvège en 2009

10 Amund B. Larsen, Kristiania Bymål. Vulgarsproget med henblikk på den utvungne dagligtale, Kristiania, Bymålslaget, 1907, p. 15.

11 Ibid.

12 Voir Karine Stjernholm, op. cit., p. 7 ainsi que Magne Bråthen et al., « Levekår på vandring. Velstand og marginalisering i Oslo ", Fafo-rapport, 5, 2007, p. 8.

13 Karine Stjernholm, op. cit., p. 39 et Magne Bråthen et al., op. cit., p. 55. 
Oslo n'est plus une ville industrielle comme on l'entendait durant la révolution du XIXe siècle, et nombreuses sont les parties de la ville qui ont depuis les années 1970 subi un processus de gentrification ${ }^{14}$. Introduit par la sociologue britannique Ruth Glass ${ }^{15}$ dans les années 1960, le concept de gentrification désigne l'amélioration physique et socioculturelle d'anciens quartiers populaires. Jonny Aspen ${ }^{16}$ soutient que l'élévation du statut des quartiers de l'est ne découle pas uniquement des changements démographiques, mais plutôt d'un discours sur la qualité du style de vie et de l'environnement urbain.

La recherche et le marquage symbolique du style de vie, de l'identité et de l'appartenance ont pris une nouvelle signification dans la culture urbaine contemporaine, ce qui implique que l'østkant à Oslo n'est plus associé à l'industrialisation et à la vie ouvrière, mais endosse une nouvelle identité progressive particulièrement visible dans les zones où la gentrification a été la plus intense. Auparavant, l'identité de l'østkant était liée à l'industrie et au statut d'ouvrier, mais aujourd'hui, habiter l'østkant s'interprète comme un choix de style de vie moderne ${ }^{17}$.

\section{Analyse}

Les deux textes sur lesquels repose notre analyse peuvent être considérés comme l'expression d'une construction identitaire, ayant comme thème la relation entre l'artiste et les quartiers est et ouest ${ }^{18}$. Nous nous intéresserons d'abord à la chanson traitant des quartiers est avant d'analyser celle portant sur les quartiers ouest. Nous résumerons et discuterons des éléments à retenir à la lumière du développement du parler d'Oslo depuis le début du XXe siècle.

\section{Don Martin et Tommy Tees, «Nilsen»}

"Nilsen " est un hommage linguistique et littéraire aux quartiers est d'Oslo. La thématique est évidente pour ceux qui connaissent l'histoire socioculturelle de la ville, mais une analyse du contenu peut également être trouvée sur https://genius. com/Don-martin-nilsen-lyrics. Le titre lui-même renvoie à de célèbres poètes de la ville tels que Rudolf Nilsen (1901-1929), Lillebjørn Nilsen (1950-) ou encore Joachim " Jokke » Nilsen (1964-2000). Le premier écrivait des poèmes sur la vie des ouvriers à l'est, le deuxième est un chanteur populaire de l'østkant tandis que le dernier était le leader convoité du groupe Jokke og Valentinerna, qui chantait en dialecte de l'østkant le côté obscur de la vie avant qu'il ne meure d'une overdose en 2000 .

14 Jonny Aspen, "Gentrifisering som kulturell diskurs », in By og byliv i endring. Studier av byrom og handlingsrom i Oslo, J. Aspen (dir.), Oslo, Scandinavian Academic Press, 2005, p. 121-150.

15 Ruth Glass, Introduction to London, Londres, Centre of Urban Studies, 1963.

16 Jonny Aspen, op. cit., p. 123.

17 Karine Stjernholm, op. cit., p. 32.

18 Pour l'intégralité des paroles, voir les annexes. 
La chanson a pour thème la division de la ville d'Oslo, qui apparaît dès la deuxième ligne, où Don Martin chante "Boka om Oslo er fortellinga om to byer " ("Le livre d'Oslo est le conte de deux villes »), faisant référence au roman $A$ tale of two cities de Charles Dickens. Dès la première ligne du texte, l'ambiance est posée : "Tunge skyer ligger over byen, som om Tor styrer " ("Des nuages lourds reposent sur la ville, comme si Tor y régnait »). Tor est un dieu de la mythologie norroise, celui qui contrôle l'orage. La division de la ville n'est donc pas présentée de façon idyllique.

La première indication linguistique d'une affiliation aux quartiers est se retrouve à la ligne 2 : "Boka om Oslo er fortellinga om to byer. " Le suffixe défini singulier $-a$ est un trait caractéristique des quartiers est. Si boka et fortellinga sont probablement des formes largement utilisées partout à Oslo, historia (" histoire») à la ligne 3 (également utilisée à la ligne 51) ressort en revanche comme une forme assez emblématique de l'est. Le choix des suffixes de définitude laisse donc peu de doute sur le fait que les paroles reflètent une nette appartenance à l'est, en plus de l'expression du genre littéraire.

La prononciation "O O lo ", où la séquence <sl> est prononcée comme une fricative post-alvéolaire, est également à interpréter comme emblématique des quartiers est. Néanmoins, cette prononciation se retrouve dans toute la ville. Toril Opsahl et Unn Røyneland ${ }^{19}$, renvoyant à Olaf $\mathrm{Broch}^{20}$, indiquent que ce trait était déjà répandu dans les milieux " plus snobes " plusieurs décennies auparavant. Ainsi, même si cette prononciation se retrouve fréquemment dans toute la ville, le maintien de la prononciation «sl » avec une réalisation alvéolaire de $\langle s\rangle$ doit être considérée comme un choix très marqué d'appartenance aux quartiers ouest.

Don Martin poursuit : "Hvis du lurer på hvem jeg rapper, det er klart hvilken" ("Si tu te demandes de qui je rappe, il est clair de qui »). Cette séquence laisse peu de doute sur l'origine du locuteur dans la ville d'Oslo, ce qui est confirmé à la ligne suivante : "Jeg er fra Oslo øst, det har du kanskje skjønt" ("Je viens d'OsloEst, ça tu l'as peut-être compris »). Avec les paroles «passet er oransje grønt» («le passeport est orange-vert »), Don Martin fait explicitement référence aux couleurs du club sportif de Romsås, une ville de banlieue située dans la circonscription de Groruddalen à la périphérie est d'Oslo. "Grønlandsleiret la hovedgata " ( Grønlandsleiret est la rue principale »). Ici, le chanteur emploie une nouvelle fois le suffixe - $a$, tout en affirmant que la véritable ville se limite à la partie est. En effet, Grønlandsleiret se voit attribuer le statut de rue principale d'Oslo, à défaut de la célèbre Karl Johan gata. Grønlandsleiret, aujourd'hui située à Grønland, reliait autrefois Gamle Oslo (littéralement « le vieil Oslo ») à Christiania (c'est-àdire le centre historique de la ville initialement appelé Oslo lorsque Christian IV

19 Toril Opsahl, Unn Røyneland, "Osloungdom : født på solsiden eller i skyggen av standardtalemålet ? ", Norsk Lingvistisk Tidsskrift, 1, 2009, p. 103.

20 Olaf Broch, "Lyden [š] som ekspressivt middel i Oslo-målet », in Fonologi/Phonology. Festskrift til Hjalmar Falk, E. Håkon Jahr et O. Lorentz (dir.), Oslo, H. Aschehoug \& Co, 1985 ; republié dans Fonologi/Phonology, Oslo, Novus, 2008, p. 146-157. 
établit Christiania en tant que capitale de la Norvège en 1624). Aujourd'hui, Oslo et Christiania ont fusionné en une seule et même ville, le nom Oslo ayant été retenu après $1924^{21}$. Grønlandsleiret appartient donc à l'est, et les quartiers ayant connu la présence danoise dans la ville sont totalement exclus de l'univers de Don Martin.

À la ligne 8, Don Martin chante "Jeg er en groruddøL som nettopp fLytta lengre inn " ( Je suis un habitant de Groruddalen qui justement a déménagé loin vers le centre »). Il indique ici son identité aux quartiers est par la réalisation flap rétroflexe du son $<\mathrm{l}>$ dans fLytta, mot dans lequel le suffixe $-a$ exprime également le prétérit du verbe.

Aux lignes 12 et 13, il chante que " Grønland blir gentrifisert som Grünerløkka, $O g$ de som bodde der nettopp blir pressa lengre ut " "Grønland s'est gentrifié tout comme Grünerløkka, et ceux qui y vivaient sont pressés loin vers l'extérieur »). Observons de nouveau l'emploi du suffixe $-a$ en tant que morphème du participe passé du verbe presse (forcer/presser). Il est clair que thématiquement, la gentrification n'est pas vue comme un phénomène positif.

Jonny Aspen ${ }^{22}$ souligne que l'utilisation du concept de gentrification en Norvège est caractérisée par un manque de réflexion critique. Dans la tradition anglaise, le terme connote assez clairement les mécanismes d'exclusion sociale et l'augmentation importante des coûts immobiliers, ce à quoi s'ajoute une opposition politique locale des populations affectées par la gentrification, un phénomène associé à l'économie mondiale et à l'exercice du pouvoir.

La gentrification entraine donc le déplacement des groupes défavorisés vers les zones urbaines périphériques. Dans la reconstruction de la ville, l'identité se module et se modifie par rapport au lieu, c'est pourquoi les acteurs les plus forts ont la mainmise sur la reconstruction ${ }^{23}$. Dans la chanson, Don Martin ne se voit pas prendre part au processus de gentrification ; pourtant les artistes ainsi que les universitaires sont les groupes les plus concernés par ce phénomène. À ce titre, nous considérons dans notre analyse le rôle de Don Martin comme significatif.

À la ligne 14, Don Martin emploie la forme sjukehus (" hôpital ") qui reflète son identité aux quartiers est, étant donné que la forme sykehus est caractéristique des quartiers ouest.

Dans le refrain, plusieurs formes sont significatives des quartiers est :

- lua (le bonnet) où le morphème - $a$ marque la forme définie singulier ;

- neri (ned $i$ [au fond]);

- øya, où le morphème - $a$ marque le pluriel de øye (œil) ;

- GamLebyn et HoLmlia, prononcés avec le <l> flap rétroflexe ;

- Oflo, de nouveau prononcé avec une assimilation de la séquence $<$ sl $>$.

21 Åse Wetås, Namneskiftet Kristiania. Oslo, Oslo, Novus, 2000.

22 Jonny Aspen, op. cit., p. 125.

23 Oddrun Sæther, Marit E. Ruud, Byen som symbolsk rom, bypolitikk, stedsdiskurser og gentrifisering $i$ Gamle Oslo, Oslo, Byggforsk, 2004, p. 76. 
Tommy Tee, qui chante aux côtés de Don Martin, énumère plusieurs localités de l'est d'Oslo, fait référence à saint Hallvard, protecteur de la ville, "du kan kalle han for Halvar» (" tu peux l'appeler Halvar »), et se pose en représentant de toute la ville d'Oslo alors qu'il renvoie exclusivement à sa partie est (" hele byen kant ti kant", " toute la ville d'un bord à l'autre ") et ne mentionne jamais les quartiers ouest.

Dans le deuxième couplet, l'emploi de la forme gutta ("le garçon ") retient notre attention. Il est à noter que cette forme pourtant fréquente n'est pas tolérée en bokmål. Quant à l'expression bull i gjerdet (" trou dans la clôture »), il aurait été attendu que Don Martin préfere la forme høl avec un abaissement vocalique, plus caractéristique de l'est. L'abaissement des voyelles ouvertes étant propre au parler de l'østkant traditionnel, ce trait semble toutefois être en récession dans le nouveau parler émergent d'Oslo, idée renforcée par Janne B. Johannessen ${ }^{24}$, qui défend que le terme de voyelles ouvertes est plus pertinent dans ce contexte, maintient que ce trait n'est plus associé au parler traditionnel de l'est. Janne B. Johannessen ajoute que l'absence de voyelles ouvertes dans le parler traditionnel de l'est se retrouve depuis les années 1970 , et que ce changement s'est produit cinquante ans auparavant. Les habitants d'Oslo restent cependant conscients que ce trait est lié aux quartiers est. Par conséquent, il aurait été attendu dans " Nilsen ", qui autant sur le fond que sur la forme constitue un hommage à l'est de la ville, que Don Martin marquerait davantage son appartenance locale à travers ce trait.

Néanmoins, il convient de souligner que la forme hull rime avec tull ("bêtise ») à la ligne précédente, ce qui pourrait justifier l'emploi de cette forme. On est quand même frappé par le fait que dans un texte dont le fond ainsi que la forme constituent un hommage à l'østkant, ce trait caractéristique de la variété est ne soit pas davantage mis en relief. Les formes expressive et poétique semblent ici entrer en conflit.

On peut également avancer cette hypothèse pour le mot d'emprunt telefon prononcé de la même façon que daktiler avec une syllabe accentuée suivie de deux syllabes non accentuées. Cette réalisation est à interpréter comme un trait du parler qui intègre le nouveau parler émergent d'Oslo (alors que ce trait semblait être en recull ${ }^{25}$ ). Dans ce contexte, la métrique semble être une explication qui détermine la prononciation : en effet, dans la forme métrique actuelle, seule l'accentuation de la première syllabe est possible. Il en va de même pour perfekt à la ligne 5 .

À la ligne 33, nous retrouvons la forme sjappa (" échoppe »), qui est rarement employée avec le suffixe -en dans le parler d'Oslo, de même que veiene ("les routes ", forme définie au pluriel avec le suffixe-ene) à la ligne 34. En norrois, ce mot est masculin, donc on s'attend pour des raisons étymologiques à ce qu'il

24 Janne B. Johannessen, "Oslo-språket de siste to hundre år ", in Talemål etter 1800. Norsk i jamforing med andre nordiske språk, H. Sandøy (dir.), Oslo, Novus, 2016, p. 277-278.

25 Voir Vanja Jahnsen, "Øst og vest for Akerselva. På vei mot ett oslomål ? ", Språklig samling, 3-4, 2002, p. 27-31. 
assume la flexion du masculin. Une recherche dans le corpus $N o T a^{26}$ démontre qu'aucune autre forme n'est utilisée.

Selon notre première hypothèse de la diffusion du morphème $-a$ dans toute la ville d'Oslo, on pourrait s'attendre à ce que le mot vei prenne le suffixe $-a$ au pluriel pour "sursignaler » l'identité, mais ce résultat semble ici infirmer notre hypothèse. La métrique peut de nouveau expliquer pourquoi l'emploi du morphème - $a$ n'est pas possible avec le mot vei : la forme veiene présente une syllabe de plus que veia, ce qui est nécessaire pour que la rime puisse se faire.

En outre, la forme $b$ Landa (" mélanger ") avec flap rétroflexe et suffixe- $a$ apparaît à la ligne 35 , de la même manière que $b$ Landinga (" le mélange ») à la ligne 36. À la ligne 37, nous relevons le suffixe - $a$ dans le nom de la rue Torggata, et à la ligne 38 un flap rétroflexe dans le mot du quartier GamLebyenn. Le mot prinsessa (« la princesse ») à la ligne 39 est relié à l'adjectif possessif au féminin $m i$, qui est la forme qui s'impose pour les substantifs féminins. Don Martin se sert ensuite du flap rétroflexe dans le mot bLokker ("HLM ») à la ligne 39, directement suivi par la forme contractée vi’kke (vil ikke [" ne veut pas »]).

À la ligne 40, on retrouve en plus du flap rétroflexe dans fLytte (déménager), la forme objet dem (eux) en forme sujet. Cette utilisation du pronom objet pour une place sujet est fréquente dans le parler classique de l'østkant, mais pas très habituelle dans le parler d'Oslo d'aujourd'hui ${ }^{27}$. Une recherche dans le corpus NoTa ne donne aucun résultat pour ham (lui) en fonction sujet, ni à l'est ni à l'ouest. On observe que Tommy Tee emploie la forme attendue de (ils) à la ligne 45. Il s'agit donc possiblement d'une variation intra-individuelle.

À la ligne 52, Don Martin utilise la forme heltemod (courage, vaillance) avec un possible voisement de $t$ à $d$. Il est difficile de se prononcer sur les raisons de cette forme. Le voisement des consonnes est caractéristique du danois, raison pour laquelle il était présent en Norvège à l'époque où le danois était utilisé à l'écrit. La norvégianisation de $d$ à $t$ à l'écrit a été l'un des changements proposés par Knud Knudsen en 1866 lors de la réforme qui s'est imposée presque partout en riksmål en 1907, et puis pour une autre liste de mots en $1917^{28}$. Dans la langue orale, il est possible que ce trait soit toujours en usage afin d'indiquer une appartenance à l'ouest. Il est donc très difficile d'expliquer pourquoi Don Martin s'en sert dans le contexte actuel. On peut spéculer que l'ancienne forme $-d$ signale quelque chose d'archaïque comme le concept de heltemot (" chevalerie ") en lui-même. Le $d$ pourrait alors renforcer le contenu du terme en question, mais il s'agit là d'une hypothèse.

26 NoTa est un corpus oral réalisé dans les années 2000, comportant 166 informateurs originaires de toute la ville d'Oslo. Il a pour but de recenser la façon de parler des habitants d'Oslo en fonction de leur sexe, âge, et formation socioculturelle/professionnelle. Pour plus d'informations, voir http://www. tekstlab.uio.no/nota/oslo/index.html.

27 Vanja Jahnsen, op. cit., p. 30.

28 Arne Torp, Lars Vikør, Hovuddrag i norsk språkhistorie, Oslo, Ad Notam, 1993. 
À la ligne 53, Don Martin prononce veit (sait) avec une diphtongaison de la voyelle du radical, initialement longue (contrairement à l'ouest où on s'attend à une monophtongue, ici vet). À la ligne 58, il chante sjøl (lui-même), qui signale l'appartenance à l'est à l'opposé de selv, une variante à la fois plus neutre et caractéristique de l'ouest.

Enfin, dans le dernier refrain, Tommy Tee ne renvoie plus aux quartiers, mais aux boîtes populaires de l'østkant à Oslo.

\section{Oslo-Ouest : "Berumsgrammatikk " par Erik et Kriss}

Le ton dans la chanson " Bærumsgrammatikk » ("La grammaire de Bærum ») est assez différent de celui de "Nilsen ». L'utilisation fréquente d'expressions argotiques en début de texte annonce une description caricaturale du vestkant, symbolisé par la ville de Bærum. C'est donc dans une optique parodique ou ironique de l'appartenance aux quartiers ouest que la chanson doit être interprétée. À ce titre, nous voulons souligner que la comparaison directe des deux chansons serait déséquilibrée, puisque "Nilsen" est un hommage à l'est, tandis que «Bærumsgrammatikk» est une description ironique de la vie dans le vestkant. Pourtant, cette distance ironique vis-à-vis de l'ouest sert également à mettre en relief la vie aisée des gens nés dans cette partie de la ville qui, pour la plupart des Osloïtes, a des connotations beaucoup plus positives que celles de l'est.

Dès le premier mot de la chanson, le morphème - $a$ est employé. Plusieurs études ont ainsi montré que ce trait était en augmentation dans l'ouest d'Oslo ${ }^{29}$.

Dans le cadre du projet TAUS (Talemålsundersøkelsen i Oslo - Investigation de la langue orale à Oslo) conduit dans les années 1970 , Western ${ }^{30}$ avait déjà comparé les informateurs les plus âgés aux plus jeunes et avait noté une utilisation croissante du suffixe $-a$ chez les jeunes. Karine Stjernholm ${ }^{31}$, qui a également étudié ce suffixe, a démontré qu'il est aujourd'hui en cours d'établissement dans l'ouest, mais que son usage n'avait pas considérablement augmenté dans l'intervalle qui sépare le corpus TAUS (1970) de NoTa (2000). En outre, l'emploi du suffixe dans «Bærumsgrammatikk » semble confirmer la neutralisation du suffixe dans l'ensemble de la ville ${ }^{32}$.

À la ligne 127, Erik et Kriss chantent que "A-ending er uhørt ", " le suffixe - $a$ ne s'entend pas ", ce qui renforce le caractère parodique du texte - voulu ou non, illustré à la ligne 128 par la phrase " hytta heter hytten", " hytta se dit hytten ". Nous trouvons par ailleurs le mot hytta (le chalet) à la ligne 151 avec le suffixe $-a$ à la forme définie du singulier. En fait, le mot hytta semble exiger le suffixe $-a$ à

29 Voir Vanja Jahnsen, op. cit., ainsi que Knut Western, "a-endinger i Oslo-mål ", Skrift nr. 5. Talemålsundersøkelsen i Oslo, 5, 1977.

30 Knut Western, op. cit.

31 Karine Stjernholm, op. cit.

32 Toril Opsahl, Unn Røyneland, op. cit., p. 95-119. 
la forme définie du singulier, tout comme le mot sjappa dans «Nilsen $»^{33}$. Remarquons que pour le substantif hytta, rien n'indique que le suffixe -en soit admis dans l'oslomål traditionnel, voire il serait probable que le suffixe -en marque le dialecte de Bergen plus que celui d'Oslo.

À la ligne 59, on trouve la forme dressjakka (la veste), qui constitue un exemple plus discutable. À Oslo, la forme jakken est admise, mais il paraît que la variante en $-a$ est assez neutre dans toute la ville. Nous supposons que si Erik et Kriss avaient voulu donner une impression encore plus vestkant, ils auraient choisi le suffixe $-e n$.

Bærum est identifié comme bygda (la bourgade), bygd faisant davantage référence à l'idée de banlieue/périphérie qu’à la notion de ville. On aurait peut-être pensé que l'opposition ville-campagne et ville-région donnerait une mauvaise connotation à Barum, mais en raison de son statut socioéconomique, les chanteurs affirment que "alt annet kalles "mad slum" ("partout ailleurs, c'est le ghetto fou ").

Un autre marqueur indiquant l'appartenance linguistique se retrouve à la ligne 5 : "her blir aldri noe rappa" ("ici on ne pique jamais rien »). De nouveau, Erik et Kriss utilisent le suffixe $-a$, cette fois pour marquer le participe passé du verbe rappe. Le verbe rappe (" piquer") constitue une variante moins soutenue que stjele ("voler »), et ce registre informel pourrait justifier l'emploi du suffixe en -a outre la rime, car rappa rime avec slapp’a (« se reposer»).

Dans l'ensemble du premier couplet, on ne retrouve pas de marqueurs linguistiques qui placent ce texte dans l'ouest d'Oslo, mais le contenu ne laisse aucun doute sur le fait que Bærum se distingue de "alt annet" (" tout le reste ") par son classement élevé sur l'échelle socioéconomique. Cette idée est d'ailleurs étayée tout au long du texte. Aux lignes 7, 8 et 9, les auteurs font référence au statut économique important des membres de la famille.

Parmi les autres traits caractéristiques des quartiers est et qui se diffusent à l'ouest, on relève à la ligne 11 le marqueur flap rétroflexe dans $h j u L$ (" roue »), de même qu'avec kLysete ("snob ") aux lignes 39, 86 et 135, bobLebad (" jacuzzi ", 105), bobLebade (106), gamLe ("vieux », 114), gLa'sport (" porte de verre », 115) et bLondine ("blonde »,118).

À la ligne 12, ils continuent en disant mobba (harcelé) avec le suffixe $-a$ au participe passé du verbe, qui est encore une fois un exemple de la popularité du suffixe $-a$ aussi à l'ouest. Plus loin à la ligne 12 , fo $\boldsymbol{L}$ (mauvais) est également prononcé avec un flap rétroflexe au lieu de l'attendu « 1 » latéral. Dans le contexte actuel, on peut supposer que le flap rétroflexe donne un effet emphatique à l'expression foL fag ("type insignifiant "), ce qui peut également être le cas pour Gutta er gLætte ("les garçons sont snobs », ligne 54).

33 Voir Helge Lødrup, «Hvor mange genus er det i Oslo-dialekten? », Maal og Minne, 103 (2), 2011, p. 120-136. 
Ce n'est qu'à la ligne 21 qu'apparaît une forme qui peut renseigner de l'appartenance d'Erik et Kriss à Oslo-Ouest : ils prononcent le verbe vet (sait) avec une monophtongue, à l'opposé de la diphtongaison de Don Martin. Erik et Kriss développent ce thème dans le couplet suivant, où ils considèrent " triste " que les fils du papa disent veit (ligne 23). Erik et Kriss ont recours à l'assimilation avec hetére (" heter det» [" dit-on »], ligne 22) et éru (" er du » [es-tu], ligne 24). Ce trait semble être fréquent partout à Oslo, et il n'est pas très marqué, surtout en contextes informels. Ce phénomène étant non marqué, il serait emblématique de l'ouest de ne pas l'appliquer, comme à la ligne 34, où Erik et Kriss chantent gir $\boldsymbol{d e g}$ (" te donne ») avec prononciation nette de $d$ et pas de $r$, ou encore plus ha det

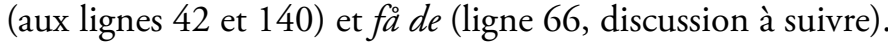

À la ligne 28, ils prononcent firesifret ("à quatre chiffres »). À l'est, on s'attendrait au suffixe $-a$. Ici, Erik et Kriss indiquent linguistiquement leur attachement au vestkant à travers le suffixe -et qui découle de la langue danoise écrite. Aux lignes 38 et 85, ils parlent des pappaguttene (les fils à papa), contrairement à Don Martin qui prononçait gutta (les garçons). Paradoxalement, Erik et Kriss utilisent aussi la forme gutta, aux lignes 54 et 74 . Cela peut indiquer que cette dernière forme est assez neutre partout dans la ville, alors que la forme en -ene signale plutôt une appartenance à l'ouest.

À la ligne 66, Erik et Kriss emploient le pronom sujet de (ils) comme forme objet, ce que Larsen ${ }^{34}$ interprète comme la tentative des classes moyennes d'imiter la langue écrite. Une forme similaire au nominatif et à l'accusatif peut aussi être interprétée comme une prolongation du système pronominal du parler traditionnel de l'est, qui inclut aussi la variante des quartiers ouest.

Aux lignes 78 et 79 , ils prononcent le nom du lieu à Bærum où ils habitent, Hofle, avec la même fusion <sl> que chez Don Martin. Comme mentionné ci-dessus, la prononciation de $<$ sl $>$ en tant que fricative alvéolaire suivie d'une latérale alvéolaire, serait un fort marqueur de l'ouest. Erik et Kriss ne vont donc pas très loin dans leur marquage linguistique de leur appartenance au vestkant. Dans leurs choix d'expression linguistique, on observe plusieurs caractéristiques qui démontrent clairement que les parlers de l'østkant et du vestkant convergent, en même temps que la position périphérique (à l'extérieur du centre-ville) des deux chanteurs joue un rôle.

À la ligne 96, Erik et Kriss accentuent le mot Kaviar à la première syllabe. Cette prononciation est inattendue pour les quartiers ouest, mais plusieurs explications s'opérant simultanément peuvent être avancées. Une première raison peut être que le duo s'attache fortement aux dialectes norvégiens parlés à l'ouest de la ville et que certaines caractéristiques de la langue norvégienne sont plus fréquentes à la périphérie que dans les quartiers du centre d'Oslo-Ouest. Une autre raison pourrait être que ce trait est moins marqué qu'auparavant, et qu'il comporte des

34 Amund B. Larsen, op. cit., p. 28-29. 
connotations autres que celles associées avec l'est, comme la masculinité, la grandeur, etc. Finalement, la métrique peut de nouveau être pertinente. Une accentuation alternative du mot n'est pas possible, étant donné la versification.

À la ligne 131, Erik et Kriss évoquent la descente des voyelles dans le parler d'Oslo en insistant sur le fait qu'il faut dire lyst (envie) et non pas lost. Ils excluent donc la variante abaissée de leur parler.

Tout comme Don Martin et Tommy Tee, Erik et Kriss mettent en relief Grønland et Grünerløkka dans leurs paroles, mais en s'en éloignant : ils estiment que "Grønland er utland og Grünerløkka ukjent " ("Grønland c'est l'étranger et Grünerlokka c'est inconnu »). Ils enchaînent en disant que "østkanten er slumland» ("les quartiers est sont des bidonvilles»).

\section{Discussion}

À travers l'analyse des paroles de deux chansons ayant comme thème la ville d'Oslo, nous avons pu dégager les principales tendances de l'évolution du parler contemporain d'Oslo.

Nous pouvons ainsi confirmer que les variétés traditionnelles de l'østkant et du vestkant ont convergé pour former une variété orale commune à toute la ville, en même temps qu'un grand nombre de traits permettent toujours de marquer son affiliation à l'est ou à l'ouest. Ces traits sont utilisés quotidiennement par les habitants d'Oslo, mais également dans des textes littéraires, musicaux ou poétiques, comme démontré dans cette analyse. Selon Toril Opsahl et Unn Røyneland ${ }^{35}$, plusieurs oppositions traditionnelles persistent entre la variété de l'est et celle de l'ouest, mais leur nombre diminue, de même qu'il est de plus en plus difficile d'associer un trait comme typique d'un quartier ou d'un autre. Cela veut dire que le parler contemporain d'Oslo donne l'impression d'être plus uniforme en comparaison avec les précédentes décennies. Les variétés de l'est et de l'ouest convergent, et ce processus va dans les deux sens. La situation peut être illustrée de la manière suivante :

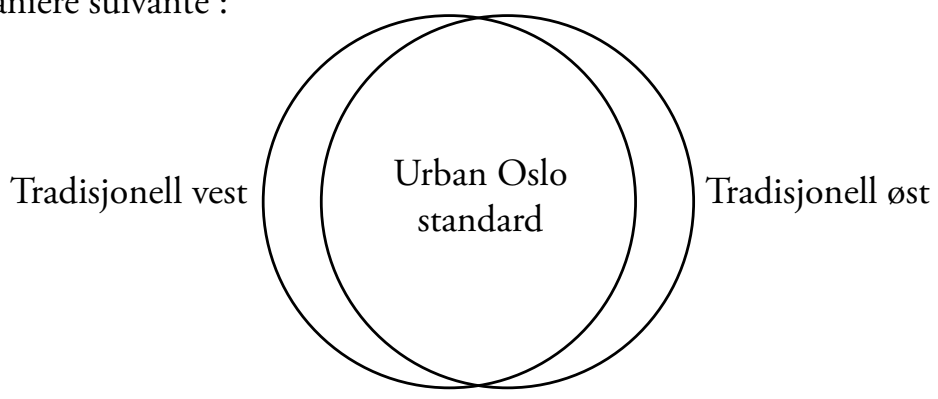

Image 3 - Les parlers de l'østkant et du vestkant convergent. Les deux variétés contribuent de manière équivalente à cette convergence.

35 Toril Opsahl, Unn Røyneland, op. cit., p. 104-105. 
Les deux variétés traditionnelles contribuent de manière équivalente à la formation d'un nouveau parler urbain à Oslo. Néanmoins, sur la base de nos précédents travaux ${ }^{36}$, cette manière de représenter les résultats de la convergence est incorrecte, car la variété de l'est a subi des pertes plus importantes que celle de l'ouest. Étant donne l'analyse présentée dans cet article, une telle conclusion peut sembler inattendue. Dans "Nilsen ", l'affiliation linguistique à l'est semble beaucoup plus prononcée que l'affinité pour l'ouest dans "Bærumsgrammatikk". Dans ce dernier, la question de la langue est soulevée, mais les choix linguistiques n'indexent pas uniquement une identité ouest, et semblent au contraire assez ouverts aux traits venant de l'est.

Même si les parlers de l'est et de l'ouest convergent, Karine Stjernholm ${ }^{37}$ souligne que la contribution n'est pas égale. Même si le parler de l'ouest a subi des pertes dans ce processus, il apparaît que la plupart des traits fréquents dans les années 1970 peuvent encore être utilisés dans le parler contemporain d'Oslo, tandis que plusieurs traits de l'est ne sont plus courants. Le corpus NoTa démontre, entre autres, que le parler de l'est ne contient plus le choix de l'infinitif variable entre $-a$ et $-e$ (kloyvd infinitiv) ni l'article devant les noms propres (han Per, « le Pierre »). Une partie importante du paradigme pronominal a disparu, y compris les formes dem/døm (eux), henne(r) (elle) ou vårs (notre), en fonction sujet aussi bien qu'en fonction objet. Les voyelles hautes descendent et le système à trois genres grammaticaux est perdu ${ }^{38}$.

Concernant le parler ouest, on peut noter que l'adjectif possessif préposé (min bror / far / mor / bil [mon frère / père ; ma mère /voiture, etc.]) est aujourd'hui archaïque, mais il n'est pas complètement tombé en désuétude, contrairement aux exemples ci-dessus.

On pourrait soutenir qu'une grande partie de la grammaire de l'est a disparu, mais que les catégories grammaticales communes entre l'est et l'ouest survivent, avec une variation provenant des deux variétés originales. Une représentation plus correcte du résultat de ce processus serait donc la suivante :

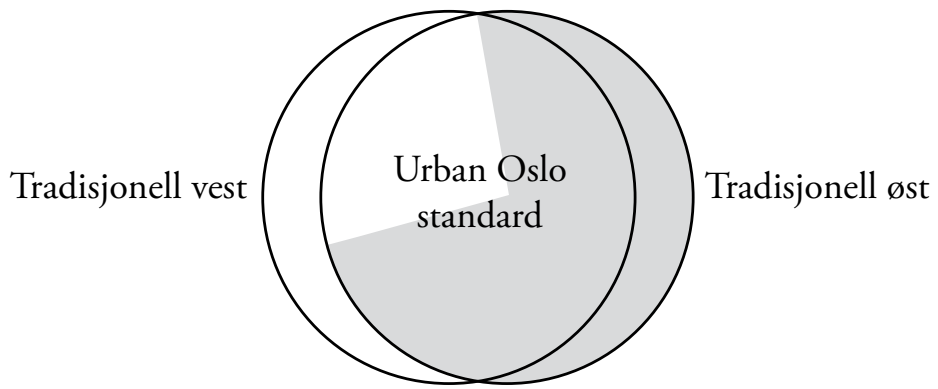

Image 4 - Le résultat de la convergence entre les parlers de l'østkant et du vestkant à Oslo (en gris, ce qui a disparu)

36 Voir Karine Stjernholm, op. cit.

37 Ibid.

38 Voir Helge Lødrup, op. cit. 
Cette figure montre que le processus de convergence a en fait donné lieu à une advergence, c'est-à-dire une fusion des deux variétés où l'une s'est imposée plus que l'autre. Les variantes de l'østkant et du vestkant n'ont donc pas contribué au même degré dans ce processus fusionnel. La partie grise illustre que la variété est a connu des pertes plus importantes. En même temps, le parler contemporain d'Oslo présente une variation importante à l'intérieur des différentes catégories grammaticales, qui peut être activement exploitée afin de signaler une appartenance, respectivement à l'est et à l'ouest, selon des motifs socioéconomiques.

Cela est illustré par la figure suivante :

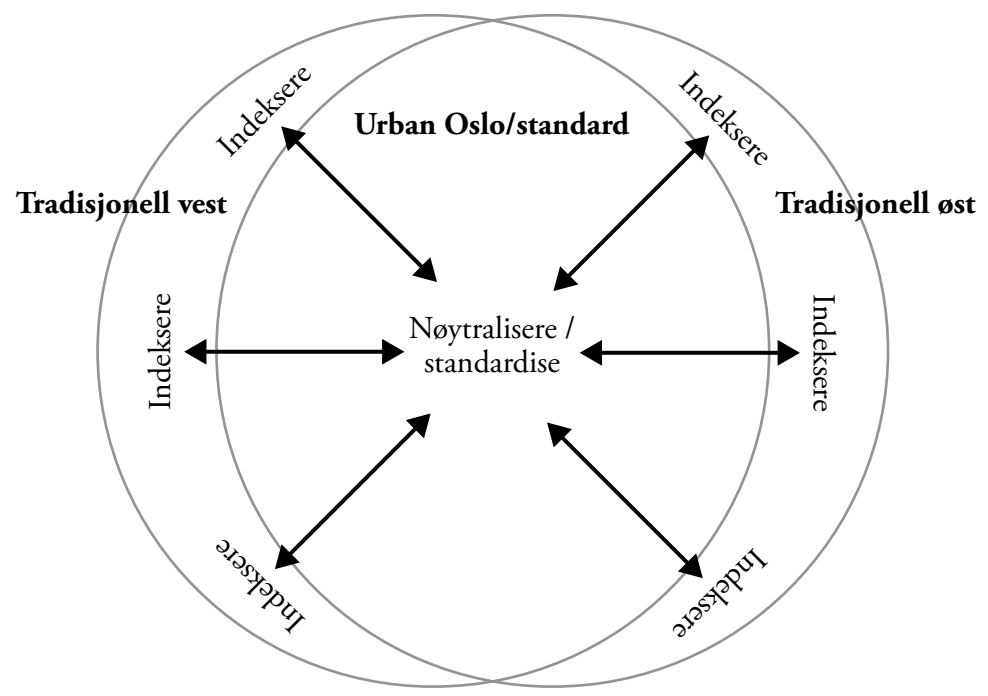

Image 5 - La situation linguistique actuelle à Oslo

Au centre de la figure 5, nous trouvons les caractéristiques linguistiques qui sont perçues comme neutres sur un axe est-ouest, tandis que les extrêmes contiennent des traits linguistiques qui peuvent être utilisés pour marquer une affiliation à l'est ou à l'ouest. La position d'un certain trait à l'intérieur ou à l'extérieur de ce centre neutre découle des facteurs individuels et contextuels. La fréquence, c'est-à-dire le nombre de traits potentiels de l'est ou de l'ouest dont les Osloïtes se servent simultanément, joue un rôle important ${ }^{39}$.

La figure indique enfin que le dialecte des habitants d'Oslo varie autour d'une partie commune assez importante située au milieu de la figure, mais que les locuteurs de l'idiome d'Oslo peuvent indexer leur appartenance au parler est ou ouest en se déplaçant vers les extrémités de la figure. Le déplacement vers le milieu de cette figure peut être interprété comme une façon de standardiser ou de neutraliser sa langue. Parallèlement, un mouvement vers les zones extérieures de la figure

39 Karine Stjernholm, op. cit., p. 154. 
pourrait être interprété comme une déviation de la norme au bénéfice du dialecte $^{40}$. À partir du modèle d'Auer, nous pouvons soutenir que l'indexation d'une affiliation ouest est un comportement dialectal qui place le parler du vestkant hors de la langue orale normée. Le parler oral standard devient la manière la plus neutre de se produire à l'oral.

Le fait que Don Martin et Tommy Tee choisissent davantage de marquer leur affiliation à l'østkant plus qu'Erik et Kriss à l'ouest peut être interprété de deux façons.

D'abord, il est évident que cette situation peut être comprise comme une réaction au fait que le parler d'østkant a accusé les pertes les plus importantes dans le nivellement entre les parlers est et ouest ${ }^{41}$. Johnstone prône que la tendance qu'ont les dialectes à se niveller "may spark the kinds of metapragmatic activities that lead to ideological differentiation among dialects $»$. Vu la nouvelle identité gentrifiée de l'østkant, il semble très probable que marquer son affiliation à l'est soit vu comme plus authentique qu'indexer une affiliation à l'ouest.

Enfin, il est possible qu'Erik et Kriss aient moins besoin de marquer une identité linguistique, car ils bénéficient déjà d'un statut de par leur appartenance à l'hégémonie traditionnelle. De ce point de vue, la distance ironique envers l'ouest donne plus de crédibilité dans l'environnement hip-hop qu'une nette identification avec les milieux aisés d'Oslo-Ouest.

Traduction de Sarah Harchaoui

40 Peter Auer et al., Dialect Change. Convergence and Divergence in European languages, Cambridge, Cambridge University Press, 2005.

41 Barbara Johnstone, op. cit., p. 391. 


\section{ANNEXES}

De l'est : Don Martin og Tommy Tee : « Nilsen »

1 Tunge skyer over sentrum som om Tor styrer

2 Boka om Oflo er fortellinga om to byer

3 Som om historia til hovedstan var Charles Dickens

4 Hvis du lurer på hvem jeg rapper, det er klart hvilken

5 Jeg fra Oflo Øst, det har du kanskje skjønt

6 Det er mitt univers, passe er oransje og grønt

7 Grønlandsleire er hovedgata i byen $\mathrm{min}$

8 Jeg er en Groruddør som nettopp frytta lengre inn

9 Vi leker ikke Queensbridge, eller South Bronx

10 Men vi har mer enn et par apekatter ibrant oss

11 Selv i en rolig by som OJloskjer det mye fucka

12 Grønland blir gentrifisert som Grünerløkka

13 Og de som bodde der nettopp blir pressa lengre ut

$14 \AA$ A bytte Aker ut mot Ahus det var sjukehus

15 Jeg er så Oflo du kan kalle meg for Nilsen

16 Rudolf, Lillebjørn, Joachim - velg hvilken

[Refrain : Tommy Tee]

17 Så kan du høre byen puste inn

18 Du finner meg me lua ne'ri øya bak i bussen din

19 Jeg er så Oflo du kan kalle meg for Halvar

20 Gamrebyn, Groru, Aker, Alna

21 Og de hører på meg overalt

22 Hormlia til Haugenstua, hele byen kant ti kant

23 Jeg er så Oflodu kan kalle meg for Nilsen

24 Så la meg sende ut en hilsen

[Verset 2 : Don Martin]

25 Du finner meg på linje 2 mot Ellingsru

26 Går av på Furuset Senter hvor gutta henger ut

27 Jeg er med folk som allti gjør noe, har noe tull på fære

28 Møt meg på Tøyenbadet sommernatt med hull i gjerde

29 Det er sjelden noe er lengre vekk enn 'telefon

30 Det er alltid noen som kjenner noen som kjenner noen

31 Av alle storbyer i verden er nok Oflominst

32 Det er her jeg føler meg hjemme, det her er byen min

33 Gutta mine er fra overalt i hele sjappa

34 Fra gutt te mann på disse veiene, og nå blitt pappa

35 Ofloer branda av alle typer folk som lever her

36 Og nettopp denne brandinga gjør byen min til det den er

37 Du finner meg i Torggata på nattesti

38 Jeg loker rundt i Gamrebyn i hatten min

39 Jeg fant prinsessa mi blant Haugenstuas brokker,

40 vi'kke frytte herfra selv om dem tilbyr meg halve kongerike

[Refrain : Tommy Tee]

41 Så kan du høre byen puste inn

42 Du finner meg med lua ne'ri øya baki bussen din

43 Jeg er så Oflodu kan kalle meg for Halvar

44 Sinsen, Disen, Kampen, Gransdaln

45 Og de hører på meg overalt

46 Mortensru ti Ammeru, hele byen kant til kant

47 Jeg er så Oflo du kan kalle meg for Nilsen

48 Så la meg sende ut en hilsen

[Verset 3 : Don Martin]

49 To tusen og én natt over Oflo øst

50 Så mange stemmer som til sammen blir til Oșlos røst

51 Som om historia den ble fortalt av Scheherazad

52 Den er om kjeltringer, om heltemod og mer om mangt

53 Og alle veit hvor jeg har hjerte mitt

54 De sier ingen særlighet skjer helt smertefritt

55 De sier byen har et hjerte som er kaldt som is
56 At allting her kan skaffes for den rette pris

57 Hvem tenker på tiggerne her og deres bør?

58 De som snakker ti seg sjøL i byen er fler enn før

59 At alt er 'perfekt her i byen har vel ingen sagt

$60 \mathrm{Og}$ det er klart at det kan blåse kaldt en vinternatt

61 Tøyen har vært Tøyen lik i hundre år

62 Det e'kke snakk om farge men om fattigdom og levekår

63 Fra de bLe sendt fra nr. 13 bort til nr. 19

64 Har dette vært en by som deler seg omtrent på midten

[Refrain : Tommy Tee]

65 Så kan du høre byen puste inn

66 Du finner meg med lua ne'ri øya bak i bussen din

67 Jeg er så Oflodu kan kalle meg for Halvar

68 Lompa, 'Barong, Beckers, GamLa

69 Og de hører på meg overalt

70 Ensjø til Ellingsrud, hele byen kant ti kant

71 Jeg er så Oflo du kan kalle meg for Nilsen

72 Så la meg sende ut en hilsen

[Outro : Don Martin \& Tommy Tee]

73 Så kan du høre byen puste ut?

74 Her er en hilsen fra en oflogutt

75 Så kan du høre byen puste ut?

76 Her er en hilsen fra en oflogutt

De l'ouest : Erik og Kriss : «Bærumsgrammatikk »

1 Bygda kalles Bærum,

2 alt annet kalles 'mad slum'.

3 Solarium er 'homsegrill' man bruker for å bli 'tanned brun'

4 'Å læxén' er å slapp' av

5 her blir aldri noe rappa

6 for alle her har sponsor, og sponsor kalles 'pappa'

7 Pappa kalles 'aksjemekler'

8 onkel kalles 'skipsreder',

9 Erik kalles 'arving' og 'fremtidi bedriftsleder'

10 Penger heter 'Mastercard',

11 hjuL kalles 'Jag',

12 er du utstøtt og mobba, er det fordi du er en ' $f æ L$ fag'

13 'Å prøve' er å ta risk'n,

14 data kalles 'harddisk'n'

15 'Visa-kort' er drinker til gode bak bardisken.

16 'Telemark' er folkesport,

17 'villa' er bosted

18 'Kragerø' er sommerspot,

19 'å chille' er å roe ned

20 bo ved pent strøk er selvfølgelighet

21 vi er bedre enn de andre, noe vi selvfølgelig vet

22 'Sørgelig' heter're når noe er leit

23 som når pappagutter ikke sier 'vet', men sier 'veit'

24 er'u 'hot' er'u heit,

25 'hannsau' er geit

26 'streit' er en ærlig kar og ærlige folk er greit

27 En 'cheap spender-bender' er en kjip gjerrig gnier

28 og firesiffret seddel kaller vi for 'Bærums-tier'

29 riktig outfit er Burberry og rosa bånd

30 og piké-skjorte fra Polo kalles 'Bærums-uniform'.

31 'Bondeland' er utmark,

32 jenter der kalles 'budeier'

33 bonde med forretningssans, det kaller vi for 'grunneier'. 
Refrain :

34 Det er Erik og Kriss som gir deg vestkantprat fra Bærum,

35 kanskje verdens beste vestkantstat

36 er'u født på beste vestkant

37 og er vestkantramp, så følg bærumsgrammatikken i vår vestkantkamp.

38 Jeg gir deg bærumsgrammatikk, sosseprat fra pappaguttene

39 kLysete eller ikke, bitch, det her er sånn vi putter det

40 om du ikke riktig digger det elle blir kvalm av're

41 kan du bare drite i det

42 det her er sånn vi skal ha det

43 Det her er bærumsgrammatikk,

44 det her er æresgrammatikk'

45 prøv å vær vår grammatikk,

46 hør og lær vår grammatikk

47 der hvor 'røyking' er astma,

48 astma er glede

49 røyking i stua

50 for 'plasma' er TV

51 Hytta er plassert på både tind og berg

52 Porschen er parkert,

53 og beltet er Lindeberg.

54 Gutta er gLætte,

55 jenter lager drama

56 hils på mine venner,

57 Dolce \& Gabbana.

58 Når jeg står borti bar'n med

59 dressjakka og slips

60 smykkene glitrer så mye, skulle tro det var trylletricks.

61 'Tolv sylinder' er bil,

62 'yacht' det er båt

63 drikken er Cristal og Don Perignon.

64 'Chicks' det er damer,

65 diamanter deres beste venn

66 smykker er tricks for å få de i seng.

67 Monaco er klassetur,

68 klassetur til Jimmis,

69 Jimmis er utested.

70 Gjest'n er 'diddy',

71 'diddy' er venner,

72 venner er kontakter.

73 'Kontrakter' er skip, og oljen som de frakter.

74 Gutta liker shopping,

75 'shopping' er ekstremsport.

76 'Klokka' er Jacob,

77 'betaling' er gullkort.

78 'Hjemme' er Hossle,

79 til Hosle vi sokner.

80 'Leilighet' er byen, og 'byen' det er Frogner.

81 Alle vil kjenne meg,

82 om de bare kunne

83 for familietreet mitt det er Forbes 400.

84 Jeg gir deg bærumsgrammatikk,

85 sosseprat fra pappaguttene

86 kLysete eller ikke bitch,

87 det her er sånn vi putter're

88 om du ikke riktig digge're

89 eller blir kvalm av're
90 kan du bare drite i det det

91 her er sånn vi skal ha det

92 Aksjer kalles 'pengelek',

93 'risk' er investering,

94 lommebok er pengesekk,

95 og 'skatt' er feil fordeling.

96 'Kaviar' er snus,

97 champis drikker vi som brus,

98 sommer heter 'utepils',

99 og ferie kalles 'cruise'.

100 Studium er 'BI',

101 medisin om du er rå-lur,

102 og sier vi 'en dag på fjord'n',

103 så mener vi 'en båttur'.

104 Tårnur er det vi stiller Rolexen etter.

105 Det er i Bærum vi har villafest til bobLebadet letter.

106 BobLebad heter 'jacuzzi',

107 eller 'fet klamydiakoker',

108 selvforsvar er 'uzi',

109 en som sliter kalles 'loker'.

110 Joker' er superkort,

111 golf kalles 'supersport',

112 og Bærum på karte kalles rett og slett for 'superspot'.

113 Drar du damer er'u 'pimp',

114 i gamLe dager het det 'dandy',

115 gLa'sport på vintern, det kaller vi for 'bandy'.

116 Handy kalles 'byggmester', (3.27)

117 helst med eget firma,

118 bLondine kalles 'sykesøster du helst skulle hatt film av' .

119 Grønland er utland og

120 Grünerløkka ukjent,

121 østkanten er slumland,

122 er du sliten er du 'utbrent'

123 utrent er 'slapp kar',

124 en dvask kar er 'chubby'

125 'kødding' er betegnelsen på tull

126 og venn er 'buddy'.

127 A-ending er uhørt,

128 'hytta' heter 'hytten'

129 jomfru kalles 'pen, urørt berte under sytten'.

130 Pånnebånd er must,

131 det heter 'lyst', ikke 'løst'

132 tørste er en tilstand som på lørdager er størst!

133 Jeg gir deg bærumsgrammatikk,

134 sosseprat fra pappaguttene

135 kLysete eller ikke, bitch,

136 det her er sånn vi putter're

137 om du ikke riktig digger're

138 elle blir kvalm av're

139 kan du bare drite i det

140 det her er sånn vi skal ha det 